\title{
New phenomena in interaction of intense ulrashort light pulses with transparent materials: from 3D self-assembled nanostructures to quill writing and nonreciprocal photosensitivity
}

\author{
Peter G. Kazansky and Martynas Beresna \\ Optoelectronics Research Centre, University of Southampton, SO17 1BJ, United Kingdom \\ pgk@orc.soton.ac.uk \\ Yasuhiko Shimotsuma and Kazuyuki Hirao \\ Department of Material Chemistry, Graduate School of Engineering, Kyoto University, Kyoto, Japan 615-8510 \\ Yuri P. Svirko \\ Department of Physics and Mathematics, University of Joensuu, FI-80101, Finland
}

\begin{abstract}
Interaction of intense ulrashort light pulses with transparent materials reveal new ultrafast phenomena. Recent demonstrations of 3D nanoripple formation, ultrafast laser calligraphy and nonreciprocal photosensitivity are reviewed.
\end{abstract}

Modification of transparent materials with ultrafast lasers has attracted considerable interest due to a wide range of applications including laser surgery, integrated optics, optical data storage, 3D micro- and nano-structuring [1].Three different types of material modifications can be induced with ultrafast laser irradiation in the bulk of a transparent material, silica glass in particular: an isotropic refractive index change (type 1); a form birefringence associated with self-assembled nanogratings and negative refractive index change (type 2) [2,3]; and a void (type 3). In fused silica the transition from type 1 to type 2 and finally to type 3 modification is observed with an increase of fluence. Recently, a remarkable phenomenon in ultrafast laser processing of transparent materials has been reported manifesting itself as a change in material modification by reversing the writing direction [4]. The phenomenon has been interpreted in terms of anisotropic plasma heating by a tilted front of the ultrashort laser pulse. Moreover a change in structural modification has been demonstrated in glass by controlling the direction of pulse front tilt, achieving a calligraphic style of laser writing which is similar in appearance to that inked with the bygone quill pen [5]. It has also been a common belief that in a homogeneous medium, the photosensitivity and corresponding light-induced material modifications do not change on the reversal of light propagation direction. More recently it have observed that in a non-centrosymmetric medium, modification of the material can be different when light propagates in opposite directions (KaYaSo effect) [6]. Non-reciprocity is produced by magnetic field (Faraday effect) and movement of the medium with respect to the direction of light propagation: parallel (Sagnac effect) or perpendicular (KaYaSo effect). Moreover a new phenomenon of ultrafast light blade, representing itself the first evidence of anisotropic sensitivity of isotropic medium to femtosecond laser radiation has been recently discovered [7]. We anticipate that the observed phenomena will open new opportunities in laser material processing, laser surgery, optical manipulation and data storage.

References

1. R. R. Gattas and E. Mazur, "Femtosecond laser micromachining in transparent materials," Nature Photonics 2, 219-225 (2008).

2. Y. Shimotsuma, P. G. Kazansky, J. Qiu and K. Hirao, "Self-organized nanogratings in glass irradiated by ultrashort light pulses," Phys. Rev. Lett. 91, 247705 (2003).

3. V. Bhardwaj, E. Simova, P. Rajeev, C. Hnatovsky, R. Taylor, D. Rayner and P. Corkum, "Optically produced arrays of planar nanostructures inside fused silica," Phys. Rev. Lett. 96, 057404-1 (2006).

4. P. G. Kazansky, W. Yang, E. Bricchi, J. Bovatsek, A. Arai, Y. Shimotsuma, K. Miura and K. Hirao, "Quill" writing with ultrashort light pulses in transparent materials," Appl. Phys. Lett. 90, 151120 (2007).

5. W. Yang, P. G. Kazansky, Y. Shimotsuma, M. Sakakura, K. Miura and K. Hirao, "Ultrashort-pulse laser calligraphy," Appl. Phys. Lett. 93, 171109 (2008).

6. W. Yang, P. G. Kazansky and Yu. P. Svirko, "Non-reciprocal ultrafast laser writing," Nature Photonics, 2, 99105 (2008).

7. P. G. Kazansky, Y. Shimotsuma, J. Qiu, W. Yang, M. Sakakura, M. Beresna, K. Miura and K. Hirao, "Ultrafast light blade: Anisotropic sensitivity of isotropic medium to femtosecond laser radiation", submitted to PRL(2009). 